\title{
Role and dynamics of matrix metalloproteinase 9 and tissue inhibitor of metalloproteinase 1 in burn patients
}

\author{
ADINA ELENA STANCIU ${ }^{1 *}$, ADINA ZAMFIR-CHIRU-ANTON ${ }^{2 *}$, \\ MARCEL MARIAN STANCIU ${ }^{3}$, MIRELA GHERGHE ${ }^{4,5^{*}}$, RAZVAN HAINAROSIE $^{6,7}$, \\ FLORENTINA LIGIA FURTUNESCU ${ }^{8}$ and DAN CRISTIAN GHEORGHE ${ }^{7,9}$
}

\begin{abstract}
${ }^{1}$ Department of Carcinogenesis and Molecular Biology, 'Prof. Dr. Alexandru Trestioreanu' Institute of Oncology, 022328 Bucharest; ${ }^{2}$ ENT Department, 'Grigore Alexandrescu' Children's Emergency Hospital, 011743 Bucharest;

${ }^{3}$ Electrical Engineering Faculty, 'Politehnica' University, 060042 Bucharest; ${ }^{4}$ Department of Nuclear Medicine,

'Prof. Dr. Alexandru Trestioreanu' Institute of Oncology, 022328 Bucharest; ${ }^{5}$ Department of Radiology, 'Carol Davila' University of Medicine and Pharmacy, 050463 Bucharest; ${ }^{6}$ ENT Department, 'Prof. Dr. Dorin Hociota' Institute of Phonoaudiology and Functional ENT and Cervicofacial Surgery, 050751 Bucharest; ${ }^{7}$ ENT Department and

${ }^{8}$ Department of Complementary Sciences, 'Carol Davila' University of Medicine and Pharmacy, 050463 Bucharest;

9ENT Department, 'Maria Sklodowska Curie' Children's Emergency Hospital, 077120 Bucharest, Romania
\end{abstract}

Received May 31, 2021; Accepted June 30, 2021

DOI: $10.3892 /$ etm.2021.10496

\begin{abstract}
Burn injuries can trigger tissue changes that can explain the variation in the level of different biochemical markers that can be recorded both locally or systemically. Some events observed in burn wounds such as vascular hyperpermeability have been associated with the release of matrix metalloproteinases (MMPs) and their tissue inhibitors (TIMPs) after trauma. Because it is unknown whether the serum levels of MMP-9 and TIMP-1 are a consequence of these destructions or a local response to thermal damage, we decided to follow their dynamics. Twenty-five patients (mean age $49.40 \pm 17.55$ years) with a total body surface area (TBSA) affected by a thermal burn of $<25 \%$ and 30 healthy
\end{abstract}

Correspondence to: Dr Adina Elena Stanciu, Department of Carcinogenesis and Molecular Biology, 'Prof. Dr. Alexandru Trestioreanu' Institute of Oncology, 252 Fundeni Street, 022328 Bucharest, Romania

E-mail: adinaelenastanciu@yahoo.com

Dr Florentina Ligia Furtunescu, Department of Complementary Sciences, 'Carol Davila' University of Medicine and Pharmacy, 37 Dionisie Lupu Street, 050463 Bucharest, Romania

E-mail: florentina.furtunescu@umfcd.ro

*Contributed equally

Abbreviations: MMP-9, matrix metalloproteinase-9; TIMP-1, tissue inhibitor of metalloproteinase-1; TBSA, total body surface area

Key words: burn injury, biomarkers, MMP-9, TIMP-1, MMP-9/TIMP-1 ratio subjects (mean age 49.70 \pm 8.04 years) were enrolled in the present study. Enzyme immunoassays were used to measure the serum levels of MMP-9 and TIMP-1. Our results showed that MMP-9 was increased 6.25-fold immediately after injury compared to the controls and remained on a plateau throughout the 7-day monitoring period. TIMP-1 showed an upward trend with an increase of $49.52 \%$ on the seventh day after triggering insult. The time-course of the MMP-9/TIMP-1 ratio followed the inverse dynamics of TIMP-1 starting from a ratio value measured at admission 3.82-fold higher than the one observed in the healthy volunteers and a highly statistically significant correlation between the values measured at different time-points during the monitoring period $(\mathrm{P}<0.001)$. The results of this retrospective study indicate that the MMP-9/TIMP-1 ratio may provide information on local changes over time, starting from the triggering insult, and may be considered as a predictive biomarker of burn evolutivity.

\section{Introduction}

Thermal injury remains a challenging disease and a leading cause of death. The analysis of the pathological lesions found in burn wounds showed an increase in vascular permeability at the site of injury (1). The responsible mechanisms are not yet well understood and can involve numerous factors (2). Some authors have described a possible role for matrix metalloproteinases (MMPs) in endothelial hyperpermeability (1,3-5). The effects of MMPs are not necessarily limited to the wound site, thus it is difficult to assess their precise role in the dynamics of the burn wound (4).

Wound healing involves complex processes that have been carefully researched over time. Some studies have described time-dependent increases in proteinase concentrations in burn wound fluid (6). High levels of proteinases (MMP-2 and MMP-9) have been found in wound fluid from chronic wounds 
but not in those produced by other types of injuries $(7,8)$. Several studies have shown that the plasmatic levels of MMP-9 are higher after burn injuries (7-10). MMP-9 appears early after burn injury, and its level rises after $48 \mathrm{~h}(9,11)$. MMP-2 can also show higher levels in burn wounds but to a lesser extent than in other injuries $(7,9)$. In burn wounds, with a typical chronic evolution, degradation products can be found but it is not clear which of the tissue proteinases are responsible for local repair (10). Another possible explanation could be the degradation of the adhesion molecules and growth factors by the local proteinases (12-14). Moreover, the expression of different tissue proteases in burn wounds could influence the prognosis (15). As a confirmation, some authors have associated increased MMP-9 concentrations with delayed healing of burned skin (16).

MMPs cannot be separated from their natural inhibitors (tissue inhibitors of metalloproteinases or TIMPs) (17). These maintain a balance that contributes to local tissue homeostasis (18-20). Independent roles for TIMPs have also been described (21). The inhibitor effect of TIMP-2 on burn-induced vascular hyperpermeability was demonstrated on some tissues, even in the presence of high MMP-9 levels (22). Serum concentrations of TIMP-1 reach a peak 2 days after a severe burn (23). TIMP-1 is known to inhibit the catalytic activity of MMP-9 in a 1:1 stoichiometric relationship (24,25).

Since the role of MMP-9 and TIMP-1 in early inflammation associated with a burn injury is poorly understood, we aimed to investigate the dynamics of MMP-9, TIMP-1, and the MMP-9/TIMP-1 ratio in the early shock phase of burn-injured patients.

\section{Patients and methods}

Patients and study protocol. This study included 25 adult patients with thermal burns (16 males/9 females, mean age $49.40 \pm 17.55$ years) admitted to the Clinical Emergency Hospital for Plastic, Reconstructive, and Burns Surgery in Bucharest, between 2018 and 2019. The study was conducted respecting the principles outlined in the Declaration of Helsinki and has been approved by the Clinical Emergency Hospital for Plastic, Reconstructive, and Burns Surgery Ethics Committee (approval no. 6627/04.10.2017).

The severity of the burn trauma is given by the burn depth and burn size. Burn size has been estimated by reference to the total body surface area (TBSA). Inclusion criteria were: i) Thermal burns with a TBSA affected by the burn of $<25 \%$; ii) deep second-degree and third-degree burns; iii) patient age over 18 years. All patients received routine treatment. They were investigated upon admission and after 2 and 7 days. Exclusion criteria were as follows: i) Patients with age under 18 years; ii) chronic heart failure or renal failure; iii) cancer; iv) primary and secondary immunodeficiency diseases; v) previously treated with systemic corticosteroids affecting the body's inflammatory response to burns; vi) treatment with doxycycline, known as an MMP inhibitor (3).

In addition, 30 healthy subjects ( 19 males/11 females, mean age $49.70 \pm 8.04$ years) were randomly selected from the individuals who volunteered for general routine health evaluation. The exclusion criteria that were applied to the patients with thermal burns were also used to select the volunteers. Written consents were obtained from patients or their legal representatives and volunteers.

Blood sample collection and processing. Blood was drawn into BD Vacutainer ${ }^{\circledR} \mathrm{SST}^{\mathrm{TM}}$ serum separation tubes purchased from Becton, Dickinson and Company as soon as possible after the admission of patients with burn wounds at the emergency department and in the following 2 and 7 days. The serum samples were obtained by blood clotting for $30 \mathrm{~min}$ at room temperature and centrifugation at 2,500 x g for $15 \mathrm{~min}$. Serum samples were then immediately aliquoted into labelled cryo-vials and frozen at $-70^{\circ} \mathrm{C}$ until analysis.

Detection of serum MMP-9 and TIMP-1 by ELISA. The quantitative determination of human MMP-9 (cat. no. DMP900) and TIMP-1 (cat. no. DTM100) was performed using ELISA kits purchased from R\&D Systems Inc. Because contamination may lead to falsely elevated serum concentrations due to the MMP-9 and TIMP-1 presence in saliva, protective measures were required to prevent contamination during the test. Three samples of known concentration were tested 20 times on one plate to assess intra-assay precision and in 40 separate assays to assess inter-assay precision. The values of the inter-assay imprecision study were similar to those of the intra-assay study with CVs ranging from 2 to $6.9 \%$. The within CVs for MMP-9 were $2.0 \%$ at a mean concentration of $83.3 \mathrm{ng} / \mathrm{ml}$ and $2.9 \%$ at a mean concentration of $1,100 \mathrm{ng} / \mathrm{ml}$ (for low- and high-concentration patient samples). CVs for the TIMP-1 were $\sim 4.5 \%$. The MMP-9/TIMP-1 ratio was calculated according to the following formula: MMP-9 (ng/ml)/TIMP-1 (ng/ml). All assays were performed in duplicate according to the manufacturers' recommendations and in such a way minimized any effects of repeated freeze-thaw cycles.

Statistical analysis. Patient data processing was performed using Microsoft Office Excel 2007 SP2 (including Data Analysis Tools). Statistical analysis was conducted using SPSS version 25 software (IBM Corp.). The difference between the serum concentrations measured in dynamics for MMP-9 and TIMP-1 was analyzed by one-way ANOVA test with Bonferroni correction, comparing their evolution during the 7-day monitoring period from the initial burn injury. The Anderson-Darling, Shapiro-Wilk, and Kolmogorov-Smirnov tests were also used to verify the data obtained after preliminary analysis and to check the consistency of the group. The correlation between investigated biomarkers was assessed using Spearman's correlation coefficient for TIMP-1 with data that did not have a normal distribution and Pearson's correlation coefficient for MMP-9 and MMP-9/TIMP-1 ratio with normally distributed data. For all tests, the significance level for statistical analysis was set at P-values $<0.05$.

\section{Results}

After a post-injury accelerated increase, MMP-9 remains at the same serum level. Data from the analysis of the sera collected from patients with burn injury, at different time intervals, compared to the healthy controls are summarized in Table I. Highly significant differences in the levels of MMP-9, TIMP-1, and MMP-9/TIMP-1 ratios were observed among patients 
Table I. Serum biomarkers in patients with skin burn injury $(\mathrm{N}=25)$ and healthy controls $(\mathrm{N}=30)$.

Patients with burn injuries

\begin{tabular}{lccccccc}
\cline { 2 - 4 } Variables & P1 & P2 & P3 & Control & P-value & P-value $^{\mathrm{c}}$ & P-value $^{\mathrm{d}}$ \\
\hline${\text { MMP-9 }(\mathrm{ng} / \mathrm{ml})^{\mathrm{a}}}^{\mathrm{a}}$ & $1,744.2 \pm 811.9$ & $1,659.6 \pm 521.4$ & $1,744.9 \pm 677.1$ & $279.3 \pm 72.4$ & $<0.001^{\mathrm{e}}$ & $<0.001^{\mathrm{e}}$ & $<0.001^{\mathrm{e}}$ \\
${\text { TIMP-1 }(\mathrm{ng} / \mathrm{ml})^{\mathrm{a}}}^{\mathrm{a}}$ & $269.9 \pm 93.6$ & $386.8 \pm 334.9$ & $534.7 \pm 381.2$ & $166.7 \pm 45.5$ & $<0.001^{\mathrm{e}}$ & $<0.001^{\mathrm{e}}$ & $<0.001^{\mathrm{e}}$ \\
MMP-9/TIMP-1 $^{\mathrm{a}}$ & $6.5 \pm 2.6$ & $5.5 \pm 2.6$ & $4.1 \pm 2.2$ & $1.7 \pm 0.3$ & $<0.001^{\mathrm{e}}$ & $<0.001^{\mathrm{e}}$ & $<0.001^{\mathrm{e}}$
\end{tabular}

${ }^{\mathrm{a} D a t a}$ are expressed as mean \pm standard deviation; ${ }^{\mathrm{b}} \mathrm{P} 1 \mathrm{vs.} \mathrm{Control;}{ }^{\mathrm{C}} \mathrm{P} 2 \mathrm{vs}$. Control; ${ }^{\mathrm{d}} \mathrm{P} 3$ vs. Control; ${ }^{\mathrm{e}}$ Statistically significant. MMP-9, matrix metalloproteinase 9; TIMP-1, tissue inhibitor of metalloproteinase-1; P1: admission; P2: 2 days after; P3: 7 days after.

with burn wounds and healthy controls. The circulating level of MMP-9 was significantly higher throughout the 7-day monitoring period compared with the healthy controls $(\mathrm{P}<0.001)$. MMP-9 increased immediately post-injury (a 6.25-fold increase compared to the controls) and remained on a plateau throughout the monitoring period as shown in Table I.

TIMP-1 is constantly increased. In contrast to MMP-9, the biomarker TIMP-1 showed a steady increase during the study period. Thus, after 2 days from burn injury TIMP-1 increased by $30.04 \%$ and after 7 days by $49.52 \%$ (Table I). On the other hand, the increase in serum TIMP-1 concentration immediately post-injury was not as high as that of MMP-9 (1.62-fold vs. 6.25-fold).

MMP-9/TIMP-1 ratio linearly decreases. Furthermore, the time course of MMP-9/TIMP-1 followed the inverse dynamics of TIMP-1, starting from a value of the ratio measured at admission 3.82-fold higher than the one observed in the healthy volunteers. The MMP-9/TIMP-1 ratio decreased during the monitoring period as follows: 2-days post-injury by $15.38 \%$ and 7 -days post-injury by $36.92 \%$.

Pearson correlations between serum levels of MMP-9 measured in dynamics. To check the consistency of the statistical analysis, a Pearson or a Spearman test was performed for consecutive harvest time. Pearson test was chosen for normally distributed data (MMP-9 and MMP-9/TIMP-1 ratio), while the Spearman test was chosen for the abnormal ones (TIMP-1). As shown in Fig. 1A and B, the serum MMP-9 concentrations measured immediately post-injury were positively correlated with the values recorded two days later $(\mathrm{r}=0.605, \mathrm{P}=0.0013)$, values which in turn were positively correlated with those measured 7 days post-injury $(\mathrm{r}=0.407, \mathrm{P}=0.04)$.

Spearman correlations between serum levels of TIMP-I measured in dynamics. The Spearman test showed a significant correlation between TIMP-1 concentrations at different times after burn injury (admission vs. 2 days post-injury: $\mathrm{r}_{\mathrm{P} 1-\mathrm{P} 2}=0.59$, $\mathrm{P}=0.0017$ and 2 days post-injury vs. 7 days post-injury: $\left.\mathrm{r}_{\mathrm{P} 2 \mathrm{P} 3}=0.39, \mathrm{P}=0.04\right)$ (Fig. 2A and $\mathrm{B}$ ).

Pearson correlations between MMP-9/TIMP-1 ratios calculated in dynamics. Furthermore, comparing the ratio of the two biomarkers measured at different time post-injury, the
Pearson's correlation coefficient was as follows: Admission vs. 2 days post-injury $\left(\mathrm{r}_{\mathrm{P} 1-\mathrm{P} 2}=0.63, \mathrm{P}=0.0006\right)$ and 2 days post-injury vs. 7 days post-injury $\left(\mathrm{r}_{\mathrm{P} 2-\mathrm{P} 3}=0.56, \mathrm{P}=0.0035\right)$ (Fig. 3A and B).

\section{Discussion}

The main findings of the present study are: i) MMP-9 increased immediately post-injury (a 6.25-fold increase compared to controls) and remained on a plateau throughout the monitoring period; ii) TIMP-1 showed a steady increase during the study period: 2 days post-injury by $30.04 \%$ and 7 days post-injury by $49.52 \%$; iii) the MMP-9/TIMP-1 ratio followed the inverse dynamics of TIMP-1 with a steady decrease: 2 days post-injury by $15.38 \%$ and 7-days post-injury by $36.92 \%$; iv) the dynamics of MMP-9, TIMP-1 and the MMP-9/TIMP-1 ratio was demonstrated by the correlation between the values measured at different time-points after burning injury.

The MMP-9 and TIMP-1 biomarkers were investigated in dynamics over 7 days. We considered that the study interval of 7 days is enough to detect both ascending and descending inflammatory responses, the 7-day period being the time interval during which the post-injury systemic inflammatory response syndrome usually occurs $(11,12,26,27)$. It should be noted that none of the patients enrolled in our study had septic complications during the monitoring period.

Burn injuries can trigger tissue changes that can explain the variation in the level of different biochemical markers that can be recorded both locally or systemically. Some events observed in burn wounds such as vascular hyperpermeability have been associated with matrix metalloproteinase (MMP) release after trauma $(1,3,6,7,9,27)$. Because it is unknown whether the serum level of MMP-9 is a consequence of these destructions or a local response to thermal damage, we decided to follow its dynamics. In line with Hästbacka et al (26), our results showed an accelerated increase in MMP-9 serum concentration immediately post-injury. Serum MMP-9 level was 6.25-fold higher than that measured for the healthy controls, probably due to the rapid release of MMP-9 from neutrophil granulocytes within minutes to hours after the triggering insult (11). In contrast to other publications $(11,23)$ describing a decrease between days 4 to 6 , in our study, after the early increase, the MMP-9 level maintained on a plateau throughout the monitoring period. If the correlation between the values measured at the patients' admission and after 2 days was highly statistically significant $(r=0.605, P=0.0013)$, 

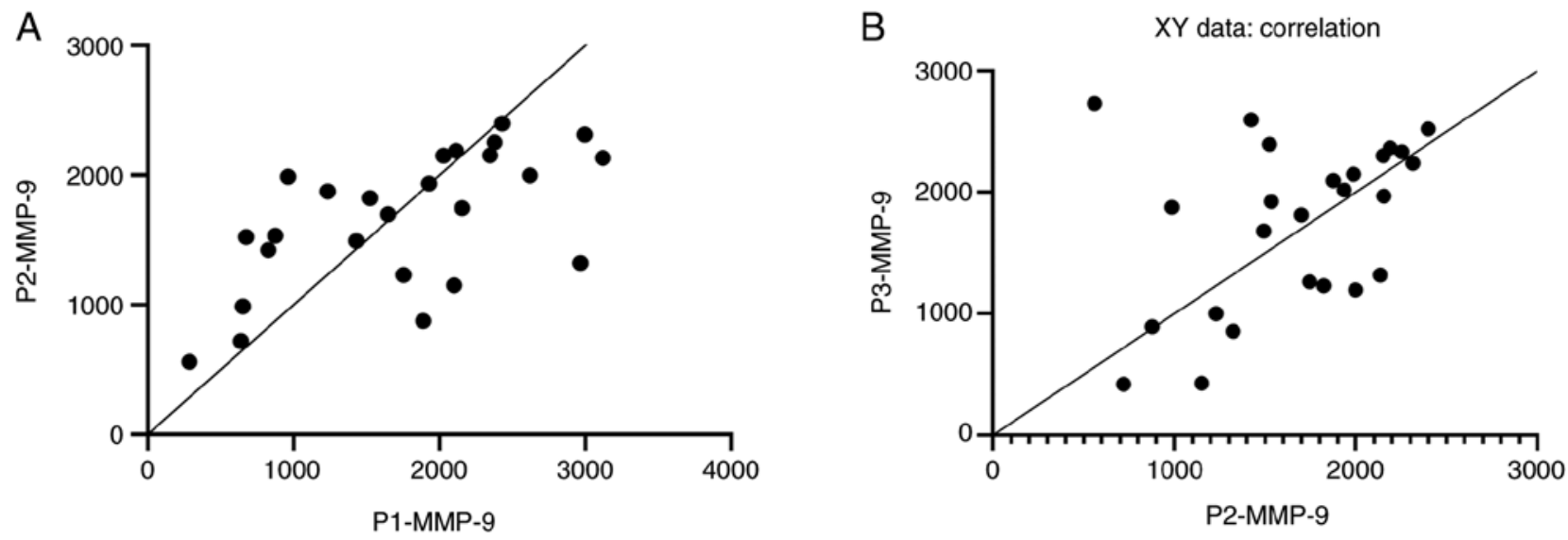

Figure 1. Pearson correlation between serum MMP-9 concentrations measured at different time-points of the monitoring period: (A) (P1: Admission; P2: 2 days after admission); (B) (P2: 2 days after admission; P3: 7 days after admission). MMP-9, matrix metalloproteinase 9.
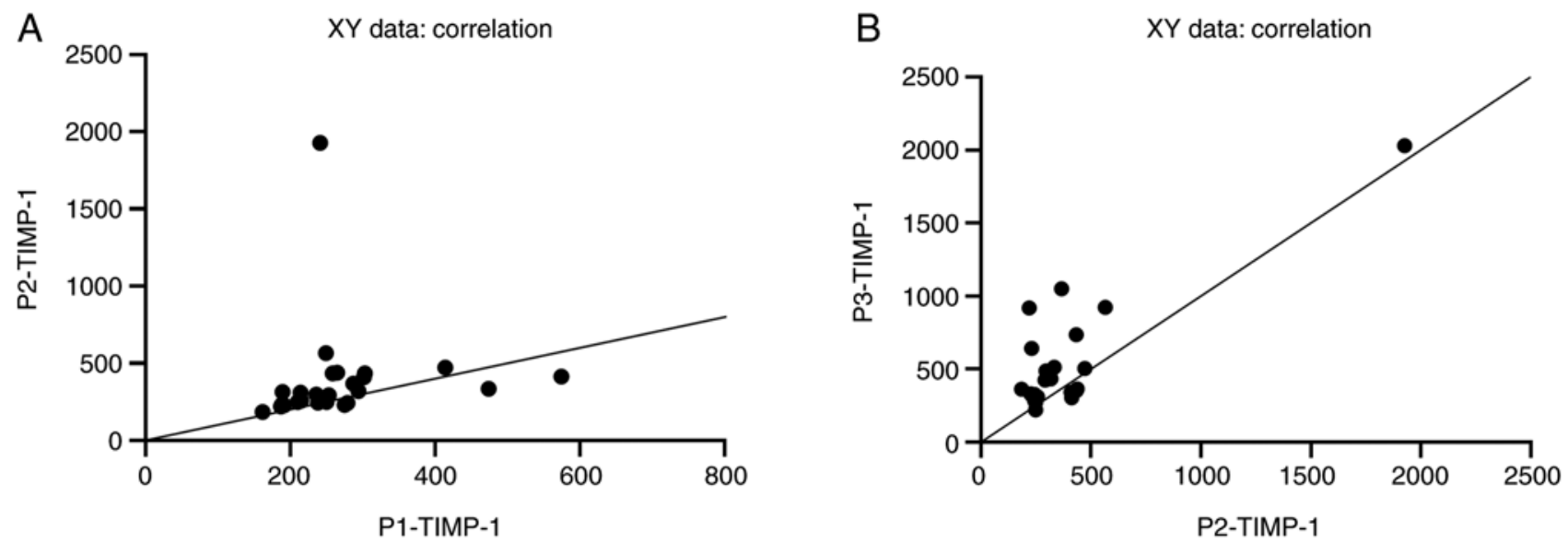

Figure 2. Spearman correlation between serum TIMP-1 concentrations measured at different time-points of the monitoring period: (A) (P1: Admission; P2: 2 days after admission); (B) (P2: 2 days after admission; P3: 7 days after admission). TIMP-1, tissue inhibitor of metalloproteinases 1.
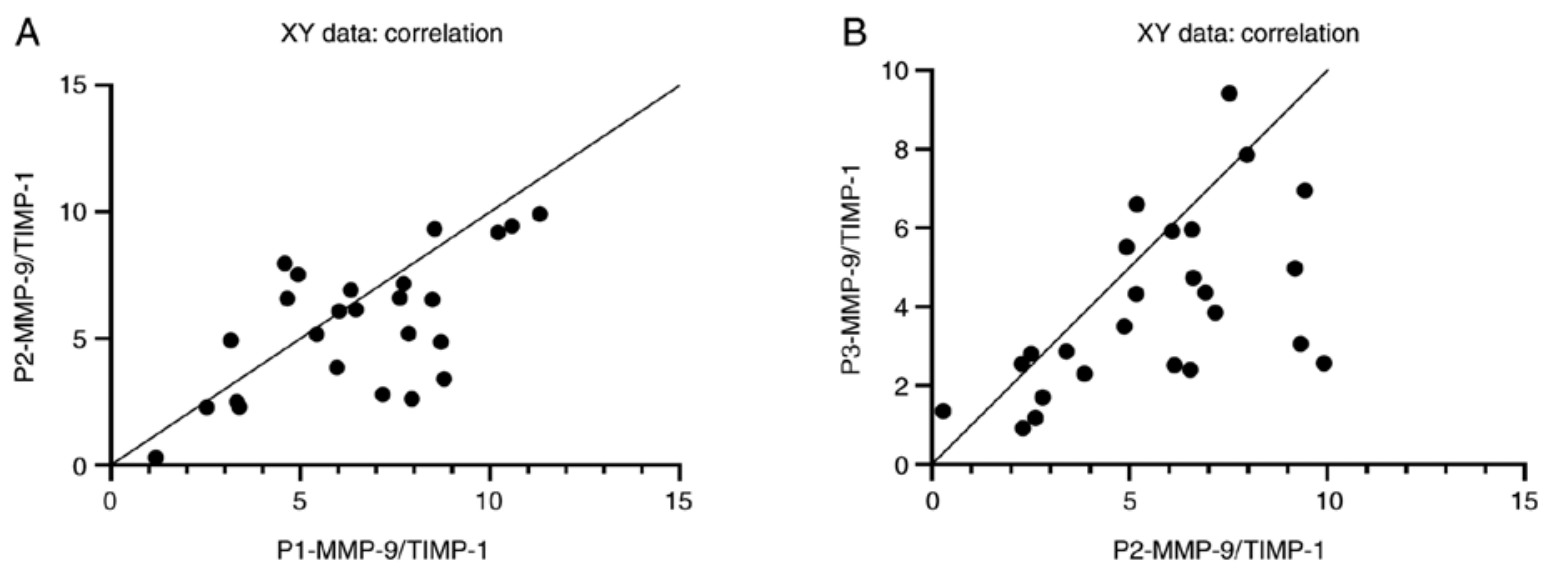

Figure 3. Pearson correlation between MMP-9/TIMP-1 ratios calculated at different time-points of the monitoring period: (A) (P1: Admission; P2: 2 days after admission); (B) (P2: 2 days after admission; P3: 7 days after admission). MMP-9, matrix metalloproteinase 9; TIMP-1, tissue inhibitor of metalloproteinases 1.

the one calculated between the measured values after 2 and 7 days did not have the same statistical power $(r=0.407$, $\mathrm{P}=0.04$ ). It is obvious, that MMP-9 does not occur as a simple local response to thermal damage. It is more than that. In burn trauma, the physiological balance is disturbed. The statistical power decrease after 7 days post-injury can be explained by the imbalance between MMP-9 and its tissue inhibitor, MMP-9 staying on a plateau, and TIMP-1 having an upward trend. Our results showing an increasing trend for TIMP-1 are in contrast to other studies $(11,23)$ that have found significantly higher 
TIMP-1 concentrations in the plasma of patients with TBSA affected by a burn of $<20 \%$ relative to healthy controls, with a median time to peak TIMP-1 concentration at 2.09 days. Given that a moderate increase in the MMP-9 serum concentration is beneficial, the epithelialization rate being partially dependent on the presence of collagen $(13,16)$, MMP-9 levels remaining constant during the 7-day monitoring period could be associated with a better-quality scar. Because none of the patients enrolled in our study had septic complications during the 7-day monitoring period, the maintaining/decrease of MMP-9 serum levels may be an indicator for better survival.

Dynamic changes in circulating levels of MMP-9 and TIMP-1 demonstrate their involvement in the early response after thermal injury. As can be seen from Table I, at all-time points the differences between serum MMP-9 and its inhibitor TIMP-1 concentrations in the study group compared to the control group were statistically significant. The time-course of the MMP-9/TIMP-1 ratio followed the inverse dynamics of TIMP-1 starting from a ratio value measured at admission 3.82-fold higher than the one observed in the healthy volunteers. The constant linear decrease in the MMP-9/TIMP-1 ratio during the 7 -day monitoring period (decrease by $14.28 \%$ in 2 days and decrease by $38.43 \%$ in 7 days from the measured value from admission) may represent an indicator of an effective healing process without hypertrophic scars and keloids for patients with a TBSA affected by the thermal burn $<25 \%$. As a confirmation, the comparison of the MMP-9/TIMP-1 ratio in dynamics showed a statistically significant correlation both between the values measured at hospitalization and those measured after 2 days $(r=0.63, P=0.0006)$ and between those measured after 2 and 7 days, respectively ( $\mathrm{r}=0.56, \mathrm{P}=0.0035)$, much better than those obtained for MMP-9 and TIMP-1 individually.

Healing processes after thermal burn evolve, as evidenced by clinical practice. The factors that influence local changes are not fully known, but our results have shown that serum proteinases may be responsible for tissue remodeling. In this regard, monitoring of the MMP-9/TIMP-1 ratio may provide information concerning local changes over time, starting from the moment of the triggering insult, ultimately indicating the need for surgery or other therapeutic approaches.

The most apparent weakness of our study is related to the small number of patients included. Despite the small sample size, three aspects need to be mentioned: i) The study group matched the control group in terms of number, sex distribution, and age; ii) all the patients included in the study had a TBSA affected by the thermal burn $<25 \%$; and iii) none of the patients enrolled in our study had septic complications during the monitoring period.

In conclusion, the results of the present study demonstrated the MMP-9 and TIMP-1 involvement in the early response after thermal injury. Our findings suggest that the MMP-9/TIMP-1 ratio may provide information on local changes over time, starting from the triggering insult, and may be considered a predictive biomarker of burn evolutivity. Further investigations are needed to confirm these findings.

\section{Acknowledgements}

Not applicable.

\section{Funding}

This research did not receive any specific grant from any funding agency in the public, commercial or not-for-profit sector.

\section{Availability of data and materials}

All data generated or analyzed during this study are included in the manuscript.

\section{Authors' contributions}

AES and AZCA conceived and planned the experiments. Experiments were performed by AES. AZCA, FLF, and MMS performed statistical analysis of the results. AES, AZCA, MMS, MG, RH, FLF and DCG contributed to the interpretation of the results. AES took the lead in writing the manuscript. All authors provided critical feedback and helped shape the research, analysis, and manuscript. All authors read and approved the final manuscript for publication.

\section{Ethics approval and consent to participate}

The study conformed to the principles outlined in the Declaration of Helsinki and was approved by the Clinical Emergency Hospital for Plastic, Reconstructive, and Burns Surgery ethics committee (approval no. 6627/04.10.2017). Written informed consent was obtained from enrolled patients or their legal representatives and volunteers.

\section{Patient consent for publication}

Not applicable.

\section{Competing interests}

The authors declare that they have no competing interests.

\section{Authors' information}

Adina Elena Stanciu: ORCID ID: https://orcid.org/ 0000-0002-9494-6686.

\section{References}

1. Wiggins-Dohlvik K, Han MS, Stagg HW, Alluri H, Shaji CA, Oakley RP, Davis ML and Tharakan B: Melatonin inhibits thermal injury-induced hyperpermeability in microvascular endothelial cells. J Trauma Acute Care Surg 77: 899-905, 2014.

2. Demling RH: The burn edema process: Current concepts. J Burn Care Rehabil 26: 207-227, 2005.

3. Stagg HW, Whaley JG, Tharakan B, Hunter FA, Jupiter D, Little DC, Davis ML, Smythe WR and Childs EW: Doxycycline attenuates burn-induced microvascular hyperpermeability. J Trauma Acute Care Surg 75: 1040-1046, 2013.

4. Rodriguez D, Morrison CJ and Overall CM: Matrix metalloproteinases: What do they not do? New substrates and biological roles identified by murine models and proteomics. Biochim Biophys Acta 1803: 39-54, 2010.

5. Pantea Stoian A, Mitrofan G, Colceag F, Suceveanu AI, Hainarosie R, Pituru S, Diaconu CC, Timofte D, Nitipir C, Poiana $\mathrm{C}$ and Serafinceanu C: Oxidative stress in diabetes. A model of complex thinking applied in medicine. Rev Chim 69: 2515-2519, 2018 
6. Young PK and Grinnell F: Metalloproteinase activation cascade after burn injury: A longitudinal analysis of the human wound environment. J Invest Dermatol 103: 660-664, 1994.

7. Wysocki AB, Staiano-Coico L and Grinnell F: Wound fluid from chronic leg ulcers contains elevated levels of metalloproteinases MMP-2 and MMP-9. J Invest Dermatol 101: 64-68, 1993.

8. McCarty SM and Percival SL: Proteases and delayed wound healing. Adv Wound Care (New Rochelle) 2: 438-447, 2013.

9. Dasu MR, Spies M, Barrow RE and Herndon DN: Matrix metalloproteinases and their tissue inhibitors in severely burned children. Wound Repair Regen 11: 177-180, 2003.

10. Lang TC, Zhao R, Kim A, Wijewardena A, Vandervord J, Xue M and Jackson CJ: A Critical update of the assessment and acute management of patients with severe burns. Adv Wound Care (New Rochelle) 8: 607-633, 2019.

11. Nagy B, Szélig L, Rendeki S, Loibl C, Rézmán B, Lantos J, Bogár L and Csontos C: Dynamic changes of matrix metalloproteinase 9 and tissue inhibitor of metalloproteinase 1 after burn injury. J Crit Care 30: 162-166, 2015.

12. Plichta JK, Holmes CJ, Gamelli RL and Radek KA: Local burn injury promotes defects in the epidermal lipid and antimicrobial peptide barriers in human autograft skin and burn margin: Implications for burn wound healing and graft survival. J Burn Care Res 38: e212-e226, 2017.

13. Guo HF, Ali RM, Hamid RA, Chang SK, Rahman MH, Zainal Z and Khaza'ai $\mathrm{H}$ : Temporal changes in the cell population and wound healing-related gene expression in deep partial-thickness burn wound model. Biomed Dermatol 4: 5, 2020.

14. Suceveanu AI, Mazilu L, Katsiki N, Parepa I, Voinea F, Pantea-Stoian A, Rizzo M, Botea F, Herlea V, Serban D and Suceveanu AP: NLRP3 inflammasome biomarker-could be the new tool for improved cardiometabolic syndrome outcome. Metabolites 10: 448, 2020.

15. Stoian AP, Razvan H, Pietrosanu C, Rusescu A, Andronache LF, Paunica S, Balalau C and Pituru TS: Modern concepts in non-surgical esthetics; a review. J Mind Med Sci 6: 190-195, 2019.

16. Simonetti O, Lucarini G, Cirioni O, Zizzi A, Orlando F, Provinciali M, Di Primio R, Giacometti A and Offidani A: Delayed wound healing in aged skin rat models after thermal injury is associated with an increased MMP-9, K6 and CD44 expression. Burns 39: 776-787, 2013.
17. Georgescu EF, Mogoantă SS, Costache A, Pârvănescu V, Totolici BD, Pătraşcu Ş and Stănescu C: The assessment of matrix metalloproteinase-9 expression and angiogenesis in colorectal cancer. Rom J Morphol Embryol 56: 1137-1144, 2015.

18. Murphy G: Tissue inhibitors of metalloproteinases. Genome Biol 12: 233, 2011

19. Raeeszadeh-Sarmazdeh M, Do LD and Hritz BG: Metalloproteinases and their inhibitors: Potential for the development of new therapeutics. Cells 9: 1313, 2020.

20. Stanciu AE, Zamfir-Chiru-Anton A, Stanciu MM, Stoian AP, Jinga V, Nitipir C, Bucur A, Pituru TS, Arsene AL, Dragoi CM, et al: Clinical significance of serum melatonin in predicting the severity of oral squamous cell carcinoma. Oncol Lett 19: 1537-1543, 2020.

21. Stetler-Stevenson WG: Tissue inhibitors of metalloproteinases in cell signaling: Metalloproteinase-independent biological activities. Sci Signal 1: re6, 2008.

22. Wiggins-Dohlvik K, Oakley RP, Han MS, Stagg HW, Alluri H, Shaji CA, Davis ML and Tharakan B: Tissue inhibitor of metalloproteinase-2 inhibits burn-induced derangements and hyperpermeability in microvascular endothelial cells. Am J Surg 211: 197-205, 2016.

23. Ulrich D, Noah EM, von Heimburg D and Pallua N: TIMP-1, MMP-2, MMP-9, and PIIINP as serum markers for skin fibrosis in patients following severe burn trauma. Plast Reconstr Surg 111: 1423-1431, 2003.

24. Stanciu AE: Cytokines in heart failure. Adv Clin Chem 93: 63-113, 2019.

25. Zurac S, Neagu M, Constantin C, Cioplea M, Nedelcu R, Bastian A, Popp C, Nichita L, Andrei R, Tebeica T, et al: Variations in the expression of TIMP1, TIMP2 and TIMP3 in cutaneous melanoma with regression and their possible function as prognostic predictors. Oncol Lett 11: 3354-3360, 2016.

26. Hästbacka J, Fredén F, Hult M, Bergquist M, Wilkman E, Vuola J, Sorsa T, Tervahartiala T and Matrix F: Matrix metalloproteinases- 8 and -9 and tissue inhibitor of metalloproteinase- 1 in burn patients. A prospective observational study. PLoS One 10: e0125918, 2015.

27. LorenteL,MartinMM,LabartaL,DiazC,Solé-ViolánJ,BlanquerJ, Orbe J, Rodriguez JA, Jimenez A, Borreguero-Leon JM, et al: Matrix metalloproteinase-9, -10 , and tissue inhibitor of matrix metalloproteinases-1 blood levels as biomarkers of severity and mortality in sepsis. Crit Care 13: R158, 2009. 